\title{
ROBUST ESTIMATION OF STATIONARY PROCESS
}

\author{
Nickolay Petrov ${ }^{1)}$, Tania Pehlivanova ${ }^{2)}$ \\ ${ }^{1)}$ U "Prof. As. Zlatarov"-Burgas, Bulgaria, Technical College nicipetrov@lycos.com \\ ${ }^{2)}$ Trakian University- Stara Zagora, Technical College - Yambol, tania_ipg@abv.bg
}

\begin{abstract}
The use of microprocessor measurement system gives the opportunity to improve the precision of the measurements. It is possible by realization of algorithms, taking in to account the changing conditions of measurement experiment. In the paper it is suggested an adaptive algorithm for calculation of the mathematical expectation $M_{X}$ in the conditions of insufficient knowledge about the interval of correlation $\tau_{C} \in\left[\tau_{C \min }, \tau_{C \max }\right]$.
\end{abstract}

Keywords: Robust estimation, random processes, precision of the measurement, stochastic measurements.

\section{INTRODUCTION}

During the practice of stochastic measurements, the unknown correlation characteristics of the random processes of the correlation interval determine the optimal interval of digitization and the necessary sample quantity [1]. The real value of the dispersion of the statistical errors $\sigma_{K B}^{2}$ while defining the mathematical expectation $M_{X}$ of stationary ergodicity accidental process $X(t)$ is determined by the following [2]:

$$
\sigma_{K B}^{2}=\frac{\sigma_{X}^{2}}{N}
$$

where $\sigma_{X}$ is the root-mean-square value of $X(t)$,

$N$ - number of the uncorrelation digital value of accidental process.

Therefore, the interval of averaging $T_{A}$ for respectively value of $\sigma_{K B}$, will be determine of equation [3]:

$$
T_{A}=N \cdot \Delta t_{D}=\frac{\sigma_{X}^{2}}{\sigma_{K B}^{2}} \cdot \Delta t_{D}
$$

where $\Delta t_{D}$ is an interval of digital.

\section{PROBLEM FORMULATION}

In values of the interval of averaging $T_{A}$ less then desired one, aren't done the requirements for precision of the measurement, and when the values are bigger it is observed unjustify decrease of the response time of the measuring process.

That determine stohastical significance for the value of $\tau_{K}$ in positioning parameters on the algorithm.

\section{PROBLEM SOLUTION}

The algorithm is based on an iterative procedure that is characterized by use of averaging in every step upon sample size. The result from the averaging of $l$-th step is determined as a function of the current time $t$, by equation (3):

$$
M_{X_{j}, l}(t)=\frac{1}{m_{l}} \cdot \sum_{s=1}^{m_{l}} X_{K}^{j}\left(t_{S}\right)
$$

where $X_{K}^{j}(t)$ is $k$-th realization of the rendam process $X(t)$, conform to $\mathrm{j}$-th measurement experiment,

$X_{K}^{j}\left(t_{S}\right)$ - member valuation of realization $X_{K}^{j}(t)$ at moment of time $t_{S}$,

$$
m_{l}=m_{l-1}+\Delta m_{l} \text {. }
$$

The procedure continues until execution of the condition (4): 


$$
\Delta M_{X_{j}, l} \leq C_{T R}
$$

where $\Delta M_{X_{j}, l}=M_{X_{j}, l}-M_{X_{j}, l-1}$

$C_{T R}$ - level of the permissible error in valuation of the mathematical expectation of the process.

Let's define probability characteristics of the iteration measurement procedure, that is function in time $t[1]$ :

- $V_{l}(t)$ - probability for execution of condition (3) till the $l$-th step;

- $P_{l}(t)$ - probability for execution of condition (3) during the $l$-th step;

- $P_{\Sigma l}(t)$ - probability for execution of condition (3) during the $l$-th step or preceding steps;

From equation (3) and (4), we receive:

$$
\begin{aligned}
& \Delta M_{X_{j}, l}(t)=\frac{1}{m_{l}} \cdot \sum_{m_{l-1}}^{m_{l}} X_{K}^{j}\left(t_{S}\right)- \\
& -\frac{\Delta m_{l}}{m_{l-1} \cdot m_{l}} \cdot \sum_{s=1}^{m_{l-1}} X_{K}^{j}\left(t_{S}\right)
\end{aligned}
$$

For large $\Delta m_{l}$, the definite properties of the sums of the random quantities are valid. Because of that, the distribution of the probability $\Delta / \mathbb{4}_{X_{j}, l}$ is near to the normal one. In case of Gaussian probability distribution of $X(t)$, the distribution of the estimates $\Delta \mathbb{1}_{X_{j}, l}$, for all magnitudes of $\Delta m_{l}$ is normal, too. That is why in the next analysis it is accepted that the probability distribution of the values $\Delta M_{X_{j}, l}$ is normal.

In case of ungaussian initial random process $X(t)$ and small $\Delta m_{l}$ the law for distribution of the probability $\Delta M_{X_{j}, l}$, could be determined, by convolution of untrivial laws of distribution [4]. It's not difficult to prove that the mathematical expectation of $\Delta M_{X_{j}, l}$ equal to zero, but the dispersion is defined as follows:

$$
D\left[\Delta M_{X_{j}, l}\right]=\frac{\Delta m_{l}}{m_{l}^{2}} \cdot \sigma_{X}^{2} \cdot\left(1+\frac{\Delta m_{l}}{m_{l-1}}\right)=\sigma_{l}^{2}
$$

where $\sigma_{X}^{2}$ is the dispersion of ungaussian random process $X(t)$.

Therefore, it can be defined the probability dension as it is shown in equation (7):

$$
W\left[\Delta M_{X_{j}, l}\right]=\frac{1}{\sigma_{l} \sqrt{2 \pi}} \cdot \exp \left[\frac{\left(\Delta M_{X_{j}, l}\right)^{2}}{2 \sigma_{l}^{2}}\right]
$$

Therefore, the probability $V_{l}(t)$ will be defined as it is shown in equation(8):

$$
V_{l}(t)=\int_{-C_{T R}}^{C_{T R}} W\left(\Delta M_{X_{j}, l}\right) d\left(\Delta M_{X_{j}, l}\right)=\Phi\left(\frac{C_{T R}}{\sigma_{b}}\right)
$$

where $\Phi(x)=\frac{2}{\sqrt{2 \pi}} \int_{0}^{x} \exp \left(-\frac{x^{2}}{2}\right) \cdot d x$ is the integral of the probability.

Respectively for the probability $P_{l}(t)$ we get:

$$
\begin{aligned}
& P_{l}(t)=V_{l}(t) \cdot \prod_{r=2}^{l-1}\left(1-V_{r}\right)= \\
& \Phi\left(\frac{C_{T R}}{\sigma_{l}}\right) \cdot \prod_{r=2}^{l-1}\left[1-\Phi\left(\frac{C_{T R}}{\sigma_{r}}\right)\right]
\end{aligned}
$$

Finally for the probability $P_{\Sigma l}(t)$ we get the equation:

$$
\begin{aligned}
& P_{\Sigma l}(t)=\sum_{i=2}^{l} P_{i}(t)= \\
& \sum_{i=2}^{l} \Phi\left(\frac{C_{T R}}{\sigma_{i}}\right) \cdot \prod_{r=2}^{l-1}\left[1-\Phi\left(\frac{C_{T R}}{\sigma_{r}}\right)\right]
\end{aligned}
$$

The obtained equation permit to calculate the average number of steps $l_{A V}$ of the iterative procedure and the characteristics of the errors of the result under fixed values of the level $C_{T R}$, the dispersion $\sigma_{X}^{2}$ and the parameter $\Delta m_{l}$.

Therefore, we get:

$$
\begin{gathered}
l_{A V}=\sum_{l=2}^{l_{\max }} l \cdot P_{l}(t) \\
P_{l}(t)=1-P_{\Sigma l_{\max }-1}
\end{gathered}
$$

where $\Delta t_{D}$ is an interval of digital.

Therefore we get for dispersion:

$$
\sigma_{K B l}^{2}=\sum_{l=2}^{l_{\max }} \sigma_{K B l}^{2} \cdot P_{l}(t)
$$


where $\sigma_{K B l}^{2}=\frac{\sigma_{X}^{2}}{m_{l}}$.

Equation (12) permits to be determined the level $C_{T R}$, necessary for assurance the needed precision of the dispersion $\sigma_{K B}^{2}$. The proposed algorithm can be used not only for defining of mathematical expectation, but for defining of all other probability characteristics of the random process $X(t)$. In the general case for the probability characteristics $\theta$ of equation (3) will be got:

$$
\theta_{X_{j}, l}(t)=\frac{1}{m_{l}} \cdot \sum_{s=1}^{m_{l}} g\left[X_{K}^{j}\left(t_{S}\right)\right]
$$

where $g\left[X_{K}^{j}\left(t_{S}\right)\right]$ is transformation in the base of the given probability characteristics $\theta$ of the random process $X(t)$. kind:

Therefore equation (4) will have the next general

$$
\Delta \theta_{X_{j}, l}=\theta_{X_{j}, l}-\theta_{X_{j}, l-1}
$$

The reasons for defining of characteristics of the errors are similary.

\section{CONCLUSIONS}

The proposed iterative procedure for equations (3) to (12) permits an increasing of the quality of the measurements in the conditions of small a priori knowledge for the properties of the random process and its probability characteristics.

\section{REFERENCES}

[1] Petrov N. Operate Reliability of Risk Technical Systems. Publishing house "Uchkov", Yambol, Bulgaria, ISBN 954-9978-26-5,2002.

[2] Cvetcov E, Basic theory of the statistical value. Peterburg, Russia, 1986

[3] Kolev N, I. Kalchev. Failure Model and Effect Analysis Applied to the Quality Control in Production. I. "Technical Ideas", vol.XXXIX, N 1-2, 2002, p.p.140-145.

[4] Petrov N., G Panajotova, M. Dulgerova. Untrivial lows of the Distribution Using for Research of the Risk Technical Systems. Collections Papers "Asen Zlatarov" University - Burgas, Bulgaria, 2003.

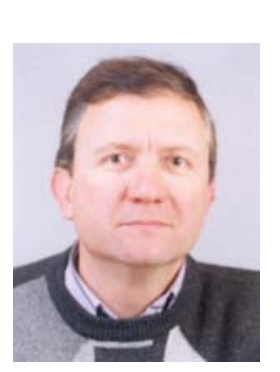

Nickolay Ivanov Petrov was born on 21 December 1953.

Education: 1972-1977 - Georgi Benkovski Higher Military Institution of Air Forces, Dolna, Mitropolia. Specialty "Communicative equipping of aircraft".

1990-1995 - Doctor's degree in Engineering, Military Academy, Institute for Perspective Defence Research, Sofia. 1999-2002 - Dr. Sc. in Engineering (Automated Systems for Information Technology And Management).

Tania Ivanova Pehlivanova was born 17.04.1965.

Education - Technical University - Sofia, Bulgaria, Department of Electrical Measurement.

Current position - assistant professor in Tracian University - Stara Zagora, Bulgaria,

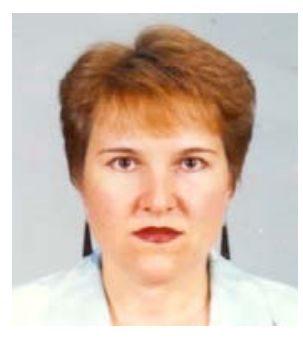
Technical College - Yambol

Areas of scientific interests - measure instruments, metrology, reliability of electrical measure instruments 